\title{
Exploring yoga as a sensory based intervention for children with sensory processing difficulties: a systematic literature review
}

\begin{abstract}
Purpose: This paper investigates the evidence of using yoga with school aged children to support their behavioural responses, self-regulation and motor abilities and their relationship between sensory processing difficulties. The aim is to determine if yoga can support individuals with sensory processing difficulties as a way to aid both sensory modulation difficulties and sensory motor disorders.

Methodology: An extensive search was conducted for studies published between 2006-2017 for review (Medline, CINAHL Plus, Scopus, AMED, PUBMED, PsycINFO, Cochrane Database of Systematic Reviews, Google scholar). In addition reference lists were manually searched for relevant studies. Duplicates and those not meeting the inclusion criteria were removed. Nine empirical studies met the criteria and were selected for this study. The reviewed literature explored the use of yoga practices including meditation, asana (poses), pranayama (breathing) techniques and/ or relaxation to support children with behaviour and emotional regulation and their motor abilities.
\end{abstract}

Findings: The reviewed literature explored the use of yoga practices including meditation, asana (poses), pranayama (breathing) techniques and/or relaxation to support children with emotional and self-regulation, behaviour, cognitive function and their motor abilities. Findings were limited yet, demonstrated how the different practices involved in yoga; postures (asana), breathing (pranayama) and relaxation offers several therapeutic tools to complement the sensory based interventions Occupational Therapists use with children with sensory processing difficulties.

Value: The results highlight the need for further research in this area with more robust designs of larger sample sizes and longer duration of intervention in order to ensure the efficacy of this practice as a sensory based intervention.

Keywords: yoga, sensory processing, children, self-regulation, behaviour, autism, ADHD, Motor abilities
Volume 2 Issue 4 - 2017

\section{Mel Campbell, Suzanne Martin}

Department of Occupational therapy, Ulster University, UK

Correspondence: Mel Campbell, Department of Occupational therapy, Ulster University, UK, Tel 0788I383750, Email melcampbellot@gmail.com

Received: October 24, 2017 | Published: November 10, 2017
Abbreviations: ADHD, attention deficit hyperactive disorder; RCT, randomised control trials; PRISMA, preferred reporting items for systematic reviews and meta-analyses; CASP, critical appraisal skills program; BUYS, big UK yoga survey; ERICA, emotion regulation index for children and adolescents; MAASA, mindful attention awareness scale in adolescents; MAIA, multidimensional assessment for interceptive awareness emotion regulation; SUDS, subjective units of disturbance scale; AASP, adult/adolescent sensory profile; ITB, imitation test battery; RSBTB, repetitive stereotype behaviour test battery; ASD, autistic spectrum disorder; $\mathrm{ABC}$, aberrant behaviour checklist; VABS, vineland adaptive behaviour scales; GRTL, get ready to learn program; CSI-4, child symptom inventory; PSPCSA, perceived competence and social acceptance for young children; ERC, emotion regulation checklist

\section{Introduction}

Latest research ${ }^{1}$ estimates that 1 in every 6 children exhibit symptoms of sensory processing difficulties which significantly impact aspects of their daily functioning, originally referred to as sensory integration dysfunction, by Occupational Therapist Jean
Ayres in the 1970's. Sensory processing is a neurological process in which sensations from the body and surrounding environment are processed, organised and integrated to enable us to give meaning to them. When sensations are processed in an organised and integrated way, the brain and nervous system assimilates them linking the mind to the body, so we can respond and act appropriately and effectively. This integration of sensory information is thought to form the basis from which we develop perceptions, behaviour and learning. ${ }^{2}$

In 1972 Jean Ayres, an Occupational Therapist developed the theory of sensory integration and its intervention, focusing her studies on the following sensory systems; vestibular, proprioceptive and tactile systems exploring how they influence behaviour and motor responses. ${ }^{3}$ Ayres's research explained the relationship between a dysfunction in interpreting sensory information with behavioural and motor development and within this, how sensory modulation and sensory motor disorders impact performance, behaviour and engagement. There is growing evidence that individuals with persuasive developmental disabilities such as autism and other diagnoses like Attention Deficit Hyperactive Disorder (ADHD) respond differently to sensory experience suggesting that some of the 
behaviours seen in these individuals overlap with sensory processing difficulties. $^{4}$

Ayres theory of sensory integration has since been adopted primarily by Occupational Therapists ${ }^{3}$ who use a sensory integration approach to provide sensory enriching experiences through the use of therapeutic opportunities and activities to help facilitate adaptive responses in order to organize the brain and enhance self-regulation. ${ }^{2}$ Although the approach of sensory integration is described $b^{2}$ as a holistic approach, which involves the whole body, the senses and the brain. Kielhofner et al. ${ }^{5}$ in 1991 criticised the theory believing that although Ayres recognised a link between the brain and body, she failed to determine the value of how sensory integration has an impact on the mind. A blended mind-brain-body therapeutic approach to sensory integration was thus proposed by Fisher et al. ${ }^{5}$ in an attempt to merge the theory of sensory integration with one which regarded the theory of the mind such as the model of human occupation..$^{5}$ hypothesised, that as individuals' develop a good neurological integration and they feel their body as a sensory motor experience, they develop selfcontrol, self-belief, self-confidence and self-esteem enabling them to interact within their environment in a more meaningful and purposeful manner.

In 2015 the theory of sensory integration and associated intervention came under scrutiny by the College of Occupational Therapy who claimed there was a lack of evidence aligned to Ayres sensory integration and other sensory based interventions and their effectiveness. ${ }^{6}$ Although the College of Occupational Therapy stated that these modalities need to be viewed with care and consideration, they acknowledged the important role Occupational Therapists play in supporting children to help manage their sensory needs and how this influences occupation performance. As the theory of sensory integration has evolved with many researchers and theorists shaping Ayres original theory, so must the sensory based interventions and techniques available to Occupational Therapists in order to reflect the contemporary times of the current population. To compliment and supplement the sensory based interventions Occupational Therapists provide, many now use alternative and complementary programs and techniques to provide enhanced sensory sensations, involve active participation and provide a meaningful and purpose activity aligned with the principals of sensory integration. ${ }^{3}$ The purpose of this literature review is to explore whether yoga is effective as a sensory based therapeutic intervention by Occupational Therapists to support children with sensory processing difficulties.

In 2016 it was estimated that over 36 million people (adults and children) practice yoga in America, ${ }^{7}$ to date there are no known figures for the UK, although a recent Big UK Yoga Survey (BUYS) has been completed by the University of Warminster with key findings yet to be published. ${ }^{8}$ This ancient practice which traditionally originated in India is fast becoming more main stream with an increasing popularity across different generations. Yoga is an embodied practice which unites the mind and body through movements in the form of asana; poses, meditation, relaxation and pranayama; breathing techniques which can improve children's physical and mental wellbeing, ${ }^{9}$ improving strength, flexibility, muscle tone and attention, memory, concentration and academic learning. ${ }^{10}$ During the physical aspects of the practice of the yoga the body moves in different directions to form poses known as asana, which provide sensory stimulation through the muscles, joints; proprioceptive, vestibular and tactile receptors to facilitate various motor responses in order for the individual to perform the poses. This interplay of sensory input to motor performance is often referred to as sensory motor. Although this sensory motor experience differs from sensory integration intervention, Ayres believed that movements of the body provides a lot of sensory input which can help organise the brain to support motor planning, body perception and coordination of two side of the body, which in turn helps the development of concentration, self-confidence, visual perception and academic learning all of which supports the process of sensory integration. ${ }^{2}$ Both Bundy and Ayres acknowledge the importance of sensory motor experience, explaining that everything rests upon a sensory motor foundation and self-esteem, self-control and selfconfidence come from feeling the body as a competent sensory motor being and from good neurological integration. Reflecting on this from a therapeutic perspective and widening the lens to seek activities to support the systems described above, yoga has emerged as an activity worthy of exploration.

A study by Parshad et al. ${ }^{11}$ identified that the condition of the mind and body are intimately interwoven and that if the mind is relaxed the muscles of the body will consequently relax. This embodied practice cultivates connections through the mind and body improving physical wellbeing whilst having a stabilising influence on the nervous system. They found that the relaxation generated by mediation helps to stabilize the autonomic nervous system, which is responsible for our heart rate. This was later supported further by Hagen I et al. ${ }^{12}$ who found that the practices of yoga can calm the heart rate and stimulate the parasympathetic nervous system which in turn has an over-riding influence on the sympathetic nervous system, responsible for the flight, fight response, often seen in children with patterns of sensory overload commonly associated with sensory processing difficulties. The practices involved in yoga such as the poses, meditation and breathing are thought to combine a shift in the physiological reaction the body may have to certain sensory stimulating situations to help calm the nervous system by stimulating the parasympathetic nervous system and altering the physiological state of the body, as well as a neuro-cognitive effect. By focusing on the breath using various pranayama; breathing and mediation techniques renewed oxygen is sent to the brain helping it to function more effectively whilst also altering the thinking patterns of the brain, influencing cognitive, behavioural and emotional regulation. ${ }^{13}$

Yoga is considered to be a versatile practice with a current interest into its therapeutic benefits for children and young people, to help them cope with the stress and challenges of everyday life, ${ }^{12}$ in addition it is a fun group activity with peers. Whilst there is growing empirical evidence on the effects of the practices of yoga on children's health and well-being, ${ }^{14}$ there is no evidence correlating this evidence specifically with the principals of sensory integration. The objective of this review was to systematically examine the evidence among selected studies of using yoga with school aged children to support their behavioural responses, self-regulation and motor abilities and specifically tease out any relationship between yoga and impact on sensory processing difficulties. Primarily, the aim is to investigate if yoga could be used as an Occupational Therapy intervention for individuals with sensory processing difficulties. 


\section{Methodology}

In order to follow the process in systematically reviewing appropriate literature for this review a structured protocol was devised and adhered to (Appendix 1) ensure appropriate studies were chosen and to minimize selection bias. The following seven electronic databases were searched for research studies, Medline, CINAHL Plus, Scopus, AMED, PUBMED, PsycINFO, Cochrane Database of Systematic Reviews, Google scholar with filters applied for articles from January 2006-January 2017. In addition, reference lists were hand searched for additional relevant studies.

The following search terms or keywords were used alone or in combination; yoga, sensory processing, children (school aged), selfregulation, behaviour, autism, ADHD, motor performance, dyspraxia, motor planning, motor skills, motor development and attention. These were adapted accordingly for each database. For the purpose of this review yoga includes a combination of breathing exercises (pranayama) physical postures (asana) and yoga nidra (relaxation). Please see (Appendix 2) for a breakdown of the database searches.

Inclusion and exclusion criteria for studies were established prior to searching the databases to aid the systematic selection of research for this review. Peer-reviewed, published manuscripts were included if they included; yoga or yoga based intervention; school aged children aged between 6-18 years; exploration of symptoms of sensory processing difficulties in the form of emotional, behavioural regulation, developmental, motor or pervasive developmental disabilities; an evaluation of emotional, behavioural regulation, cognitive or motor performance at both pre intervention and post intervention; Research designs included were randomised control trials (RCT) and quasi-experimental studies. RCT are considered the 'gold standard' in clinical research search, ${ }^{15}$ as they provide quantitative evidence to measure the efficiency of therapeutic intervention. Only articles written in English were included. Studies were excluded if they didn't meet the above criteria and were published prior to 2006, yoga was not the primarily form of intervention and articles which were dissertations, comments, letters, abstracts, guidelines, book chapters and unpublished manuscripts such as university thesis and dissertations.

An initial search retrieved a total of 861 hits, which were recorded and screened against the inclusion and inclusion criteria, after 168 duplicates were removed. Titles and abstracts were screened to establish that a total of 39 studies met the inclusion criteria. These were retrieved for full paper critique to ratify they met inclusion and exclusion criteria. This resulted in nine empirical research studies being selected for the review, author name beside each citation. ${ }^{16-24}$ For the nine final studies selected the Critical Appraisal Skills Program (CASP 2013) was used to evaluate the qualitative and quantitative methodologies of each of the studies.

Following confirmation of study inclusion in the review, an adapted version of Preferred Reporting Items for Systematic Reviews and Meta-Analyses (PRISMA) was used to identify, select and evaluate appropriate research and to collate and analyses the data from the nine studies that are included in this review, please refer to Figure 1 for details. PRISMA is considered to be a preferred method of reporting systematic reviews. ${ }^{15}$

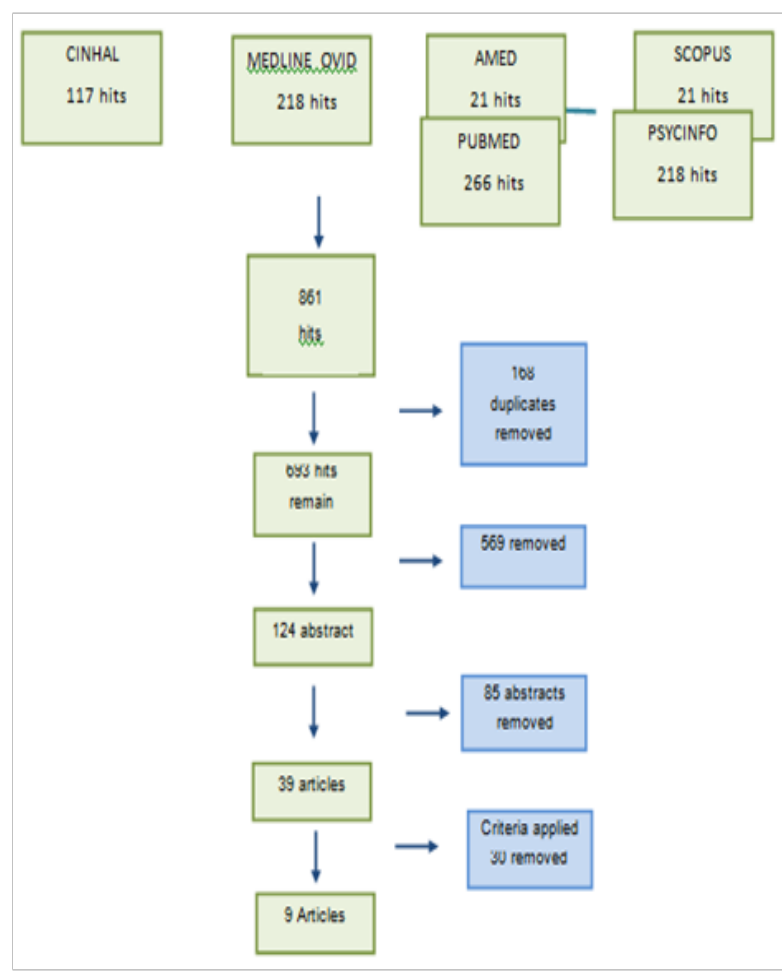

Figure I PRISMA flowchart of study retrieval for systematic review.

\section{Results}

The nine included studies were critiqued in terms of methods, intervention and quality of the study. The quality of the studies varied, the studies conducted ${ }^{16,19,21-24}$ by were deemed to be a good quality $(\geq 7)$ based on the Critical Appraisal Skills Program (CASP 2013) analysis of reviewed studies (Appendix 3) whereas those by Radhakrishna $\mathrm{S}$ et al. ${ }^{17}$ and $^{20}$ were considered to be moderate $(<7)$ and ${ }^{18}$ of a poor quality $(\leq 4)$

Six out of the nine studies stated they had gained ethical approval for their research. ${ }^{19-24}$ All of the included nine studies ${ }^{16-24}$ used purposive or convenience sampling, due to the nature of the intervention, which involved participating in the practice of yoga, blinding in the studies was not possible which was acknowledged in all of the studies. The sample size of the participants in the studies varied from six to seventy five with a total number of 338 participants across the nine studies, with ages varying from five to eighteen years. Just over $55 \%$ ( 5 of 9) of the studies had control groups. It is noted that a smaller sample size number and the absence of a control group can dilute or bias research reducing the reliability of the results and this was taken into account when considering and evaluating the findings.

In the studies the yoga sessions varied in intensity from one session in total using a pranayama technique; Mukha Bhaastrika ${ }^{18}$ or relaxation technique; yoga nidra ${ }^{19}$ or two sessions of yoga ${ }^{21}$ to twice a day yoga sessions for eight weeks ${ }^{24}$ and sixteen weeks, ${ }^{20}$ weekly sessions for sixteen weeks ${ }^{22}$ and eighteen months, ${ }^{17}$ twice a week over a period eight weeks ${ }^{16}$ twelve weeks ${ }^{23}$ and sixteen weeks. The duration of each 
class fluctuated from twenty minutes to fifty minutes with over $55 \%$ of the classes averaging forty five minutes. There was one drop-out reported in one particular study ${ }^{24}$ as a result of personal circumstances and in another study, ${ }^{21}$ twelve participants out of the seventy five were noted to attend only one out of the two yoga sessions.

Six of the studies observed the effects of yoga practice on children's emotional and behavioural regulation ${ }^{16,17,19,20,22,24}$ with two of these studies focusing on the impact of yoga on attention among children with the diagnosis of $\mathrm{ADHD}^{16,24}$ and two studies determining the effects of yoga on the behaviour of children with ASD. ${ }^{17,20}$ The other studies focused on the effect of yoga with children on their motor abilities. Please refer to Appendix 4 to view the data extraction of the studies. For the purpose of this review, a thematic approach has been adopted to organize and assimilate and synthesise the qualitative and quantitative data findings across the nine studies to establish themes. The four main themes which were apparent across the nine studies fell into the following categories; behaviour, cognitive function, emotional and self-regulation and motor abilities.

\section{Emotional and self-regulation}

Three of the studies ${ }^{19,21,22}$ investigated the therapeutic effects of yoga on emotional and self-regulation with school aged children through quantitative and qualitative measures. The number of participants varied from seven ${ }^{19}$ to $75 .{ }^{21}$ In the study by Bhavanani AB et al. ${ }^{18}$ it was focused specifically on the practice of yoga nidra, a form of relaxation with children who were classified as having disruptive behaviour as a result of their specific diagnosis. Questionnaires were employed both pre and post intervention to obtain data and scale their behaviour, anxiety and self-esteem using standardised measures. Each of the participants were exposed to 15 minutes of yoga nidra which had been progressively built up over a 13 week period from an initial 30 seconds to ensure that the participants could engage in this practice for the purpose of the study. Their breathing patterns and respiratory effort were measured at specific times during the practice by placing respiratory bands around both their chest and abdominal regions to measure the movements of these regions as a consequence of the breathing process during the yoga nidra. Although the results indicated that despite the participants exhibiting unstable patterns of breathing at the beginning of the yoga nidra practice which improved during and up to 5 minutes after the practice, the small sample size and the insubstantial control group, consisting of 6 participants of a slightly older age, was considered to provide a weak comparison and thus limitations of this study. There were no significant changes recorded post-intervention with the anxiety and self-esteem inventories.

In contrast the studies by Re P et al. ${ }^{21} \&$ Daly LA et al. ${ }^{22}$ explored the relationship between yoga and emotional regulation amongst adolescents, by analysing the effective of a yoga practice which comprised of postures, breathing, relaxation and mediation techniques of either a 40 minute practice three times a week for 16 weeks ${ }^{22}$ or at least two 50 minutes sessions. ${ }^{21}$ The study by Daly LA et al. ${ }^{22}$ evaluated evidence from a sample size of 38 students aged between 15-17 years old, who were randomly allocated to either the yoga group or the PE group which acted as the control group, whereas ${ }^{21}$ used a convenience sample of 75 mental health patients with no control group. Both of these studies collated quantitative data using a variety of different standardised self-evaluation questionnaires to record any differences in emotional regulation, disturbances and interceptive awareness, which were completed by the participants. Anecdotal qualitative data from participants was also recorded by $\mathrm{Re} \mathrm{P}$ et al. ${ }^{21}$

Daly et al. ${ }^{22}$ attempted to combine the various self- assessments Emotion Regulation Index for Children and Adolescents (ERICA), Mindful Attention Awareness Scale in Adolescents (MAASA) and the Multidimensional Assessment for Interceptive Awareness (MAIA) completed by the participants with a teachers and parent Emotion Regulation Checklist (ERC). All questionnaires were completed beforehand, at the mid-point and two weeks post intervention. Physiological measures were recorded pre and post intervention for each session $i^{21}$ study to supply further quantitative evidence in addition to the psychometric assessment for of the Subjective Units of Disturbance Scale (SUDS) and the Adult/Adolescent Sensory Profile (AASP) self-assessment form. Although ${ }^{22}$ document in their study physiological and attention measures were taken pre and two weeks post intervention in line with their self-evaluation questionnaires as mentioned above, neither the physiological and attention measures nor the Emotion Regulation Checklist (ERC) were used in the final analyses. Due to these limitations coupled with the small sample size of this particular study and the subjective nature of the students completing the self-assessments forms, the final results of the ${ }^{22}$ study should be interpreted with caution. Although, the completion of the SUDS and the AASP by the participants pre and post intervention in the ${ }^{21}$ study could be viewed with similar bias, the objective quantifiable physiological measures of pulse rates and the subjective qualitative recordings in this study show positive results, however, the restriction of two yoga sessions and the shortcomings of only determining the immediate effects of yoga post intervention rather than any short or long-term effects limit this study.

\section{Behaviour}

Radhakrishna, Koenig et al. ${ }^{17,20}$ each considered the effectiveness of yoga on children with ASD analysing changes in maladaptive behaviours and comparing it to the control groups. In both studies qualitative data of behavioural changes were targeted under the following themes; body posture, sitting tolerance, body awareness, balance, imitation skills and self-stimulatory behaviours. These studies differed in terms of the duration of the intervention and the quantitative data collection. Whereas ${ }^{17}$ based their intervention over a 5 hours of weekly integrated yoga approach which comprised of yoga asana and breathing exercise which took place over a period of two years covering two 10-month academic years with a two month gap during the summer months, ${ }^{20}$ used a daily yoga program during the school day of 15-20 minutes over a 16 week period.

Radhakrishna S et al. ${ }^{17}$ used a sample size of six students who were selected based on the inclusion criteria from a sample of fortytwo children. The intervention was on a one-to-one basis with an instructor questionnaires were completed by the parents and special educators. Parents participated in the integrated yoga therapy sessions and were trained to guide their child through what was described as being of firm guidance. Qualitative evidence identified improvements with social interaction and eye contact, motor skills for instance body posture, body awareness, sitting tolerance, balance and the ability to 
imitate as well as positive changes in maladaptive behaviours such as self-stimulatory and self-injurious behaviours. Pre, mid and post intervention quantitative information on participants behaviour was gathered through several point rating scales; the Imitation Test Battery (ITB), Repetitive Stereotype Behaviour Test Battery (RSBTB) and the Autism Institute Research diagnostic checklist form which addressed specific social-communicative behaviours. Although positive findings were recorded in this study of changes in the behaviour of children with Autistic Spectrum Disorder (ASD) following the intervention of yoga, specific limitations regarding the sample size, the reliability of the data collected by parents and observers plus parents bias needs to be considered.

To monitor changes in behaviour ${ }^{20}$ used various standardised measures the Aberrant Behaviour Checklist (ABC) and Vineland Adaptive Behaviour Scales (VABS). These provided quantitative and contextual information regarding the student's behaviour across different domains which included; social, communication, motor and daily living as identified by their parents and teacher. Forty-eight participants were selected for this study based on their diagnosis and were randomly selected to either participate in their regular morning activity or a morning daily yoga routine based on the Get Ready To Learn program (GRTL) comprising of breathing exercises (pranayama), physical postures (asanas), deep relaxation (yoga nidra) as well as some chanting over a period of 16 weeks. Outcomes in this study revealed that those whom participated in the GRTL program showed statistical significant reduction in behaviours relative to the control group in areas of irritability, agitation, crying, lethargy, social withdrawal, stereotypical behaviours, hyperactivity, non-compliance and inappropriate speech as rated by the classroom teachers. Whereas the parent Vineland Adaptive Behaviour Scales (VABS) checklist revealed an increase in the same behaviours for the sample group post intervention apart from a reduction in inappropriate speech. The authors explain that although the findings by the parents show very little significant positive effects following the yoga program, they initially scored the students behaviour more positive than the teachers and subscales were scored higher pre-test for the intervention group compared to the control group. The limitations of the study include that the teachers were not blinded to the intervention and that only $77 \%$ of parents of the participants completed the post-test checklist which may bias the findings and thus be responsible for the observed outcomes.

\section{Cognitive function}

Abadi MS, Chou CC et al. ${ }^{16,24}$ investigated the efficacy of the practice of yoga has on children with Attention Deficit Hyperactivity Disorder (ADHD), by noting any changes in their behaviour and cognitive functions such as impulsivity, inattention, self-control, hyperactivity and anxiety symptoms often associated with ADHD. Both studies had control and intervention groups comprising of twenty ${ }^{16}$ and twenty five,${ }^{24}$ although one of the participants withdrew from the intervention of the ${ }^{24}$ study leaving a total of twenty four. Each intervention group met twice weekly over eight weeks and completed 40 minutes ${ }^{16}$ or 45 minutes $^{24}$ of a yoga program. Chou CC et al. ${ }^{24}$ reported that post intervention participants of this group showed statistical change on the attention and hyperactivity sub-scales of the Child Symptom Inventory (CSI-4) compared to the control group. A significant difference was noted on the hyperactivity and impulsive scale with a reduction in fidgeting behaviour and interruptions and a rise in the ability to attend to tasks, play and academic work as documented by both parents and teachers. However, there was a discrepancy between a child's ability to remain seated when asked to do so by parents and school. Varying expectations from home and school were thought to account for this. The results of this study aligned with those by Chou $\mathrm{CC}$ et al. ${ }^{24}$ which revealed improvements in the sustained attention and discrimination function of children with ADHD, when measured using the Visual Pursuit Test and the Determination Test. Blinding protocols in either of the studies was not clear and ${ }^{24}$ did not explain the test reliability or the standardised recommended time restraints when re-administering the assessments post intervention.

\section{Motor abilities}

Of the nine studies the two studies ${ }^{18,23}$ which explored the effectiveness of yoga on motor abilities, neither had control groups. The practice of yoga varied from focusing on one session of Mukha Bhastrika, ${ }^{18}$ a bellow type of breath exercise to ${ }^{23}$ implementing twelve weeks of twice weekly 45 minutes of a yoga program consisting of poses, breathing, relaxation and meditation. Bhavanani $\mathrm{AB}^{18}$ used convenience sampling to select the 34 participants out of 63 mentally challenged students who were identified as fitting the inclusion criteria as they were able to perform the Mukha Bhastrika ${ }^{18}$ breathing technique and had received weekly yoga for the past 2-3 years. The participants engaged in nine rounds of Mukha Bhastrika ${ }^{18}$ breathing and visual and auditory reaction times were recorded using a specifically designed piece of apparatus both prior and post intervention. The quantitative data signified a statistical faster reaction time in both visual and auditory immediately post intervention for all participants. Although this study shows an improvement with reactions times, the accidental selection of the sample group and no control group poses questions as to the reliability of this particular study. Similarly ${ }^{23}$ had no control group when investigating the effects of yoga on the motor abilities of children 6-8 years. This study was characteristic of a quasiexperimental mixed method in it intentional selection of the sample group of 16 children. One of which had a diagnosis of autism the others had no known disabilities. A combination of quantitative and qualitative data was collected pre and post the 12 weeks of twice weekly 45 minutes of yoga. Quantitative measures were recorded using the Bruininks-Oseretsky Test of Motor Proficiency, the flexibility test from the sit and reach Eurofit test, the Pictorial Scale of Perceived Competence and Social Acceptance for Young Children (PSPCSA). Qualitative data from questionnaires and semi-structured interviews with parents and teachers plus anecdotal information of the participants' self-perception were recorded. Positive quantifiable findings were recorded in some motor and physical abilities such as; dynamic and static balance, running speed, agility, strength and flexibility and responses from the children, teacher and parents showed improvements in participants' behaviour, concentration, selfperception and well-being. However, only half the parents returned the questionnaires, the answers of which in the parents' perception the children were more active, relaxed, calm and showed improvements in their posture and breathing. These interpretations could be conceived as bias which with the small sample group, restricted intervention time and the lack of control group contributes to the limitations of this particular study.

\section{Discussion and conclusion}

This review systematically investigated the literature on yoga with school-aged children and the impact this has on their behaviour, cognitive function, emotional and self-regulation and motor abilities 
with the aim to explore the correlation this may have with sensory processing difficulties in order to determine if yoga could be a valid Occupational Therapy therapeutic tool to support individuals with sensory processing difficulties. Initially 39 studies were identified in which yoga was taught to a varied population of school aged children in various settings. However, after systematic critique of these studies only nine met the inclusion criteria of children aged between 6-18 years, who exhibited symptoms of sensory processing difficulties in the form of emotional, behavioural regulation, developmental, motor or pervasive developmental disabilities. Qualitative and quantitative data from these studies was synthesized in order to explore the efficiency of yoga on emotional, behavioural regulation, cognitive or motor performance.

Of the nine studies analysed, three studies explored the efficiency of yoga on school-aged children's emotional and self-regulation ${ }^{19,21,22}$ observed the impact yoga has on the sensory processing patterns of mental health adolescents and the therapeutic effects this practice can have on sensory regulation by reducing stress and anxiety. These results were also observed by Daly LA et al. ${ }^{22}$ who noted a correlation between the increases of body awareness with emotional regulation. Jensen PS $^{19}$ observed how the practice of yoga nidra; relaxation supported the breathing patterns of children with disruptive behaviour and discussed the influence this could have on the nervous system to support relaxation and induce changes in anxiety and disruptive behaviours. In this study it explains references that the flight or fight responses of the sympathetic division of the autonomic nervous system is often overly activated in children with disruptive behaviour. ${ }^{2.5}$ This component of the nervous system serves to prepare the body in response to it feeling threatened and is known to be active during periods of stress. These studies indicate that the practice of yoga can enhance the activities of the parasympathetic nervous system and thus potentially influence emotional and self-regulation.

Behaviour, attention and motor responses are considered to be the physical manifestations of the integration of sensory information received through various receptors in the body through the nervous system. When there is a dysfunction in the way this information is processed and organized, an individual may experience difficulties with movement, learning new skills and managing their own emotional regulation and ordinary sensory demands can be misinterpreted resulting in an individual feeling overwhelmed, anxious and stressed. The means in which sensory processing occurs in the nervous system is often manifested through the activity of the autonomic nervous system which is often reflected in the behaviour of the individuals who have difficulties modulating the sensory input that they receive through the nervous system. ${ }^{3}$ For example if an individual has difficulties processing tactile sensory information and is over reactive to certain sensations within this domain the sympathetic component of the autonomic nervous system will become activated. Using a sensory integration approach Occupational Therapists will often use deep pressure as a means to promote the activity of the parasympathetic nervous system and bring about equanimity to the body. Yoga has been shown to influence the actions of the autonomic nervous system, reducing anxiety induced physiological states, it is possible therefore that yoga could support individuals who exhibit heightened levels of arousal and help shift emotional regulation in children with sensory modulation difficulties.

Individuals with autism are thought to react differently to sensory stimulation to those without this diagnosis and there is a broad spectrum of literature cited $i^{26}$ which suggests that individuals with autism experience sensory modulation difficulties. In $2001^{27}$ identified that individuals with autism who are over reactive to sensory stimuli and have traits of sensory modulation difficulties display behaviours which are characteristic of an activated sympathetic nervous system. In the studies by Radhakrishna S et al. ${ }^{17}$ and, ${ }^{20}$ both demonstrated that the practice of yoga can help reduce maladaptive behaviours exhibited in children with autism, reporting a significant decrease in behaviours such as irritability, agitation and self-stimulatory behaviours with an increase in social and communicative behaviours such as compliance, eye gaze, receptive and imitation skills amongst the participants.

Abadi MS et al. ${ }^{16}$ observed changes in cognitive function amongst children with ADHD showing positive changes in areas such as attention to tasks and play activity, avoidance with learning tasks, fidgeting and impulsiveness. Similar findings were also recognized by Chou $\mathrm{CC}$ et al. ${ }^{24}$ which showed an overall improvement with attention of children with ADHD. Chou CC et al. ${ }^{24}$ explained that the practice of yoga is found to influence the activities of the autonomic nervous system by activating the parasympathetic nervous system and restoring the body in to a state of equilibrium helping to promote attention, concentration, self-control and stress management.

Yoga is considered to provide sensory input through the primary tactile, vestibular and proprioceptive neurological pathways, ${ }^{28}$ as it involves moving through a composition of motor skills to perform the poses. These sensory systems are considered to be fundamental to sensory integration in order to develop the higher functions of balance, coordination, motor planning, attention, emotional stability and visual perception with the by-products of concentration, selfcontrol and academic learning. ${ }^{2}$ This theory is supported by Chou CC et al. ${ }^{24}$ who regarded the posture control and the combination of motor skills involved when performing the yoga poses activates the nervous system in such a way to improve attention and cognitive functioning of children with ADHD. A pyramid of learning devised by Williams MS et al. ${ }^{29}$ supports this idea by demonstrating that by targeting the underlying sensory processing modalities changes in behaviour as well as academic learning can be elicited. This was notion was later supported by Lane SJ, et al. ${ }^{30}$ who provided further evidence to support the role sensory motor activities play in enhancing the processing of information in the nervous system to supply a foundation for learning and the prerequisite skills required to influence this.

The theory of sensory integration is primarily aimed at remediating the processes within the nervous system rather than using a structured method to teach new skills. During the practice of yoga motor responses are elicited through altered muscle tone and movement, by stimulating the various underlying sensory-motor pathways as identified by Cuomo N. ${ }^{28}$ Children are thought to learn about their bodies through the sensory feedback which is produced through movement and it is a key component of child development. This interplay between sensory input and motor responses through the engagement of active movement helps to develop skills. This construction of sensory motor processes is thought to also promote the higher functioning of the central nervous system which involves mental and social performance. ${ }^{2}$ The studies by Bhavanani $A B$ et al. ${ }^{18,23}$ demonstrated improved motor responses ${ }^{23}$ in balance, strength and flexibility and reaction times, ${ }^{18}$ who considers that changes on the reaction times could be induced by improved states of arousal, improved concentration or and the ability to modulate sensory input as a result of improved processing within the central nervous system. 
Occupational Therapists frequently use complementary and alternative interventions to complement the work they do with children with sensory processing difficulties. Although some of them may differ from sensory integration in terms of their application, many align with the theory of sensory integration. One of the key features of Occupational Therapy is about occupation and the under pinning components to support individuals to develop skills in order for them to perform everyday tasks and assume daily roles in their lives and sensory integration is considered to be a factor in performance. ${ }^{3}$ From the literature analysed in this review it is evident yoga provides sensory motor experience and supports the integration of mind and body connection through the central nervous system to support cognitive function, emotional and self-regulation, behaviour and motor skills. It provides a purposeful and meaningful activity which not only supports the underlying foundations to develop skills through a fun based and playful activity for children but also their overall mental and emotional well-being. Yoga is embodied practice attuning an individual's to sensations outside of their body through the muscles, joints and sense of where the body is in space to the interceptive sensations connecting the mind and body providing individuals with tools to support self-management and self-regulation. From the literature gathered in this review, it could be hypothesised that the practice of yoga is to be considered a worthy therapeutic tool to complement the sensory based interventions Occupational Therapists use with children with sensory processing difficulties. However, from the current small sample of studies available and the limitations of the studies discussed in terms of lack of control groups and small sample size to further verify the practices of yoga with this particular population further research using randomized control trials (RCT) need to be employed with larger sample groups to look at the long-term efficacy of this practice with children with sensory processing difficulties.

\section{Acknowledgements}

None.

\section{Conflict of interest}

The author declares there is no conflict of interest.

\section{References}

1. Sasson B, Carter AS, Briggs GMJ. Sensory over-responsivity in elementary school: prevalence and social-emotional correlates. $J$ Abnorm Child Psychol. 2009;37(5):705-716.

2. Ayres AJ. Sensory Integration and the Child 2nd ed. USA: Western Psychological Services; 2005. $211 \mathrm{p}$.

3. Bundy A, Lane S, Murray E. Sensory Integration theory \& practice. 2nd ed. USA: FA Davis Company; 2002.

4. Roley SS, Blanche EL, Schaff RC. Understanding the nature of Sensory Integration with diverse populations. USA: Pro Ed; 2001. 64 p.

5. Kielhofner G, Fisher AG. Mind-body relationships. In: Fisher AG, et al editors. Sensory Integration theory \& practice. USA: FA Davis Company 1991. p. 30-45.

6. Sensory integration. Practice briefing, College of occupational therapy, England: COT; 2015. p. 1-11.
7. Anon. Yoga in America. Yoga Journal \& Yoga Alliance, USA: IPSOS; 2016.

8. Anon. Big UK Yoga Study (BUYS) Science and Technology. University of Warminster; 2017.

9. Galantino ML, Galbavy R, Quinn L. Therapeutic effects of yoga for children: a systematic review of the literature. Pediatr Phys Ther. 2008;20(1):66-80.

10. Hariprasad VR, Arasappa R, Varambally S, et al. Feasibility and efficacy of yoga as an add-on intervention in attention deficit-hyperactivity disorder: An exploratory study. Indian J Psychiatry. 2013;55(S3):S379-384.

11. Parshad O. Role of yoga in stress management. West Indian Med J. 2004;53(3):191-194

12. Hagen I, Nayar US. Yoga for children and young people's mental health and well-being: research review and reflections on the mental health potentials of yoga. Front Psychiatry. 2014;5(35).

13. Gard T, Noggle JJ, Park CL, et al. Potential self-regulatory mechanisms of yoga for psychological health. Front Hum Neurosci. 2014;8:770.

14. Komitor JB, Adamson E. The complete Idiots guide to Yoga with Kids. England: Penguin Books Ltd; 2000.

15. Aveyard H. Doing a Literature Review in Health and Social Care 3rded. Berkshire: Open University Press; 2014.

16. Abadi MS, Madgaonkar J, Venkatesan S. Effect of yoga on children with attention deficit/hyperactivity disorder. Psychological Studies. 2008;53(2):154-159.

17. Radhakrishna $\mathrm{S}$, Nagarathna R, Nagendra HR. Integrated approach to yoga therapy and autism spectrum disorders. $J$ Ayurveda Integr Med. 2010;1(2):120-124

18. Bhavanani $\mathrm{AB}$, Ramanathan $\mathrm{M}, \mathrm{Kt} \mathrm{H}$. Immediate effect of Mukha Bhastrika (A bellow type pranayama) on reaction time in mentally challenged adolescents. Indian J Physiol Pharmacol. 2010;56(2):174-180.

19. Jensen PS, Stevens PJ, Kenny DT. Respiratory patterns in students enrolled in schools for disruptive behaviour before, during and after yoga Nidra relaxation. Journal of Child and Family Studies. 2012;21(4):667-681.

20. Koenig KP, Reen BA, Garg S. Efficacy of the get ready to learn yoga program among children with autism spectrum disorders: a pretest-posttest control group design. Am J Occup Ther. 2012;66(5):538-546.

21. Re P, Connell JW, Reidinger G, et al. Effects of yoga on patients in an adolescent mental health hospital and the relationship between those effects and the patients' sensory-processing patterns. J Child Adolesc Psychiatr Nurs. 2014;27(4):175-182.

22. Daly LA, Haden SC, Hagins M, et al. Yoga and emotion regulation in high school students: a randomised controlled trial. Evid Based Complement Alternat Med. 2015. p. 1-8.

23. Folleto JC, Pereira KR, Valentini NC. The effects of yoga practice in school physical education on children's motor abilities and social behaviour. Int $J$ Yoga. 2016;9(2):156-162.

24. Chou CC, Huang CJ. Effects of an 8-week yoga program on sustained attention and discrimination function in children with attention deficit hyperactivity disorder. Peer J. 2017;5:e2883.

25. Beauchaine TP, Katkin ES, Strassberg Z, et al. Disinhibitory psychopathology in male adolescents: Discriminating conduct disorder from attention-deficit/hyperactivity disorder through concurrent assessment of multiple autonomic states. J Abnorm Psychol. 2011;110(4):610-624. 
26. Brown NB, Dunn W. Relationship between context and sensory processing in children with autism. Am J Occup Ther. 2010;64(3):474-483.

27. Miller L, Anzalone M, Lane S, et al. Concept evolution in sensory integration: a proposed nosology for diagnosis. AJOT. 2007;61(2):135140 .

28. Cuomo N. Yoga with a Sensory Integrative Approach: Integrated Yoga. 1st ed. USA: Jessica Kingsley Publishers; 2007.
29. Williams MS, Shellenberger S. How does your engine run? A leader's guide to the alert program for self-regulation. USA: Therapy Works Inc; 1996.

30. Lane SJ, Schaaf RC. Examining the neuroscience evidence for sensorydriven neuroplasicity: implications for sensory-based occupational therapy for children and adolescents. Am J Occup Ther. 2010;64(3):375-390. 